\title{
Technical note: optical extinction coefficient of polydisperse spherical gold nanoparticles in water
}

\author{
Jia Cherng Chong ${ }^{a, *}$, Siew Ling Lee ${ }^{b}$, Noriah Bidin ${ }^{a}$ \\ a Laser Centre, Universiti Teknologi Malaysia, 81310, UTM Johor Bahru, Johor, Malaysia \\ ${ }^{b}$ Center for Sustainable Nanomaterials, Ibnu Sina Institute for Scientific and Industrial Research, Universiti Teknologi Malaysia, 81310 UTM Skudai, \\ Johor, Malaysia \\ * Corresponding author: andrew.chong89@gmail.com
}

\section{Article history}

Received 28 August 2016

Accepted 30 November 2016

\section{Graphical abstract}

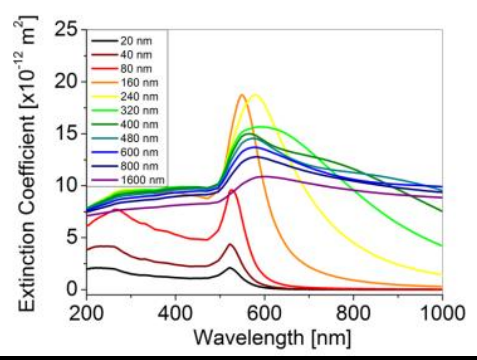

\section{Abstract}

The extinction coefficients of polydispersed gold nanoparticles up to $\sigma=360 \mathrm{~nm}$ were computed via exact solution of Mie theory. A narrow extinction peak around $520 \mathrm{~nm}$ occurs for mean particle sizes $<\mathrm{d}>$ within dipole approximation limit. Spectral characteristics for extinction coefficient computed based on increasing mean particle sizes, degree of polydispersity, composition ratio of bimodal size distributions and changes in dispersant temperature are compared. As mean particle sizes increases, the plasmon resonance peak red-shifts and broadens skewing towards infrared. Increasing polydispersity on mean particle diameter beyond dipole approximation limit decreases peak extinction coefficient values. Increasing temperature from ambient to boiling changes the peak extinction coefficient intensity value by an order of $10^{-13}$ while resonance wavelength remains unchanged.

Keywords: Mie theory, extinction coefficient, gold nanoparticles, polydisperse

(C) 2016 Penerbit UTM Press. All rights reserved

\section{INTRODUCTION}

The optical property of water dispersed gold nanoparticles has been exploited since mid 1990s for its promising applications such as biomolecular sensors [1-2] and photothermal cancer therapy [3-4]. In most cases, the usage of aforementioned gold nanoparticles (AuNPs) exploits a nanoscale physical property called localised surface plasmon resonance (LSPR) where incoming electric field component of optical radiation is strongly absorbed by in-phase oscillation of free electrons in Drude metals of size significantly smaller than the wavelength of incident beam. The resonance frequency of surface plasmons in nanosized gold is highly dependent on particle diameter and corresponds to dramatic peak shifts across experimental UV-vis extinction. Since applications of AuNPs demands LSPR response from narrow band of light waves, this makes size monitoring during and post-fabrication an upmost priority. By solving Maxwell's equations for electromagnetic radiation interacting with small conducting spheres of wavelength-dependent dielectric constant as bulk gold, Gustav Mie [5] applied a boundary condition which leads to multipole oscillations for the extinction cross-section of AuNPs. In this study, we simulate extinction coefficients of spherical AuNPs at optical frequencies on different particle size distributions (PSD). The extinction spectra presented are analyzed for future reference that is particularly useful with regards to real-time studies with PSD of citrate-based colloidal gold during synthesis.

\section{METHODOLOGY}

By expanding a series of interacting E-fields into partial waves, the exact solution of Mie theory enabled the computation of the extinction and scattering cross-section following notation described by Bohren and Huffman [6]:

$$
\begin{aligned}
& \sigma_{\text {ext }}=\frac{2 \pi}{|k|^{2}} \sum_{L=1}^{\infty}(2 L+1) \cdot \operatorname{Re}\left\{a_{L}+b_{L}\right\} \\
& \sigma_{s c a}=\frac{2 \pi}{|k|^{2}} \sum_{L=1}^{\infty}(2 L+1) \cdot\left(\left|a_{L}\right|^{2}+\left|b_{L}\right|^{2}\right)
\end{aligned}
$$

where the parameter $\mathrm{aL}$ and $\mathrm{bL}$ are given by:

$$
\begin{aligned}
& a_{L}=\frac{m \psi_{L}(m x) \psi_{L}^{\prime}(x)-\psi_{L}^{\prime}(m x) \psi_{L}(x)}{m \psi_{L}(m x) \eta_{L}^{\prime}(x)-\psi_{L}^{\prime}(m x) \eta_{L}(x)} \\
& b_{L}=\frac{\psi_{L}(m x) \psi_{L}^{\prime}(x)-m \psi_{L}^{\prime}(m x) \psi_{L}(x)}{\psi_{L}(m x) \eta_{L}^{\prime}(x)-m \psi_{L}^{\prime}(m x) \eta_{L}(x)}
\end{aligned}
$$

Parameter $m$ is the ratio between frequency dependent complex refractive index of gold, $\mathrm{n}$ and real refractive index of the surrounding medium, nm such that $m=n / n m$. In equation 1 (a) and $1(b)$, the diffraction parameter, $\mathrm{x}$ is given expressed in terms of wave-vector, $\mathrm{k}$ such that $x=2 \mid \mathrm{k} / / \mathrm{d}$ with $\mathrm{d}$ as the diameter of AuNP. The absorption cross-section is simply $\sigma_{\text {abs }}=\sigma_{\text {ext }}-\sigma_{\text {sca. }}$. For equation 2(a) and 2(b), $\psi_{\mathrm{L}}$ and $\eta_{\mathrm{L}}$ are Ricatti-Bessel cylindrical functions [7] where the prime indicates derivative. $\mathrm{L}$ is the order of partial waves involved in computation where $\mathrm{L}=1$ correspond to dipole plasmon oscillation and $\mathrm{L}=2$ being quadrupole oscillation and so on. 

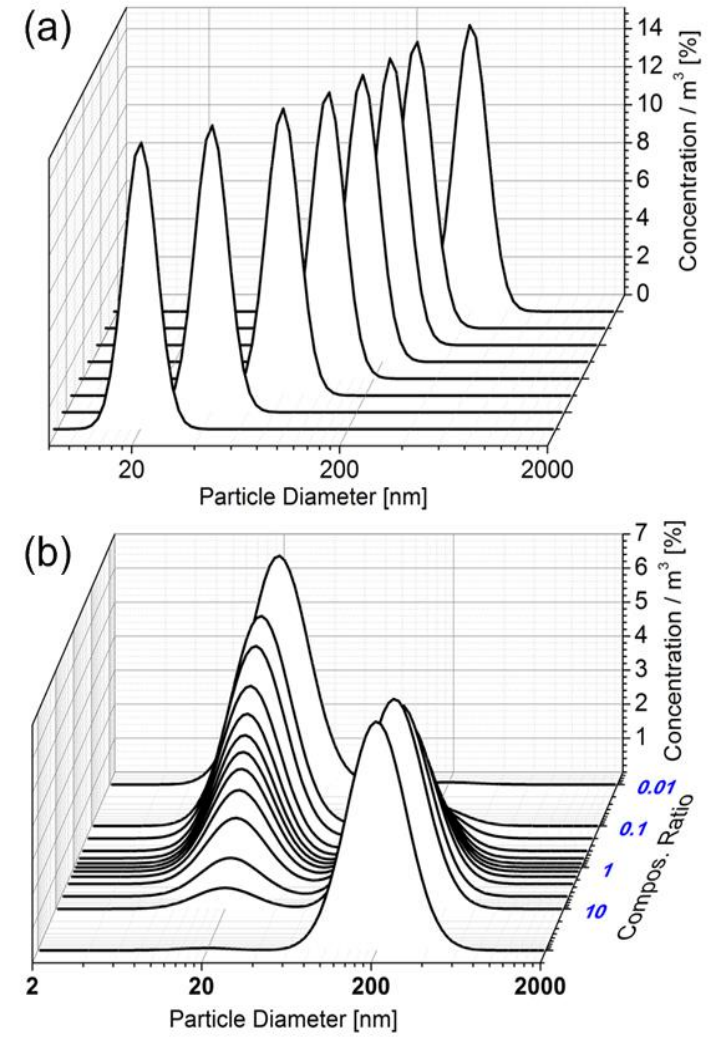

Fig. 1 (a) Size distribution used for computing changes in optical extinction spectra with respect to changing mode particle sizes with $\sigma=$ $40 \mathrm{~nm}$. (b) Changing composition ratio, $y$ for bimodal particle sizes of 200 $\mathrm{nm}$ and $20 \mathrm{~nm}$, both peaks with $\sigma=100 \mathrm{~nm}$.

To simulate realistic colloidal systems, we consider dispersed AuNP population taking log-normal PSD function frequently used to describe colloidal and gas aggregated nanoparticle samples as:

$$
f_{\log }\left(\frac{d}{2}\right)=\frac{1}{\sqrt{2 \pi} \ln (\beta)} \exp \left(-\frac{\left[\ln \left(\frac{d}{2}\right)-\ln \left(\frac{d_{0}}{2}\right)\right]^{2}}{2[\ln (\beta)]^{2}}\right)
$$

where $\beta$ indicates the bin width of the log-normal function; if the particle sizes are monodisperse enough, it is difficult to distinguish lognormal and normal distributions. For multimodal PSDs, the extinction coefficient is the sum of extinctions contributed by the number concentration of each particle sizes such that:

$$
\sigma_{\text {ext }}=\sum_{i=1}^{n} \sigma_{i} N_{i}
$$

Four sets of PSD have been used to simulate extinction coefficient based on realistic colloidal systems with spherical particles by exact solution of Mie theory in equation 1. Extinction coefficient spectra corresponding to monomodal PSD from $\langle\mathrm{d}\rangle=20 \mathrm{~nm}$ to $400 \mathrm{~nm}$ was calculated at a standard deviation of $40 \mathrm{~nm}$ as in figure 1 (a). The computation extends into polydisperse particles with $\langle\mathrm{d}\rangle=20 \mathrm{~nm}$ and $60 \mathrm{~nm}$ by increasing standard deviation from $\sigma=10 \mathrm{~nm}$ up to $\sigma=360$ nm. Bimodal PSD computations are carried out with varying concentration ratio between two particle populations with $\gamma=\left\langle\mathrm{d}_{200}\right\rangle$ : $\left\langle\mathrm{d}_{20}\right\rangle$ as in figure 1 (b). All results are simulated with input of complex refractive index of gold by Johnson and Christy [8] and real refractive index of water at $25^{\circ} \mathrm{C}$ at wavelength resolution of 500 steps spanning $200 \mathrm{~nm}$ to $1000 \mathrm{~nm}$. Temperature changes of the dispersant are investigated using Segelstein model [9] with increment of $10^{\circ} \mathrm{C}$ in each step.

\section{RESULTS AND DISCUSSION}

Similar to the case of confined particle in an infinite potential well, electronic energy levels for nanostructured gold become increasingly discrete. This effect on energy levels is most obvious for semiconductors where bandgap energy increases with smaller diameter and the beginning of the threshold bandgap transition is shifted to higher energies [10]. In metallic nanoparticles, quantum size effects exist but much subtler compared to semiconductors. In order to observe the localization of energy levels, AuNPs must be of size lesser than 2 $\mathrm{nm}$ as the spacing of energy level must exceed thermal energy at 0.026 $\mathrm{eV}$. This is due to the conduction band in metals is half filled and its density of energy levels is so high that a noticeable separation of energy levels within the conduction band can only be observed when the system is made up of a few hundreds of atoms. AuNPs at $10 \mathrm{~nm}$ in diameter would have energy level spacing of only $0.167 \mathrm{meV}$. Experiments has found that the interband transition for bandgap between the lowest unoccupied molecular orbital (LUMO) and the highest occupied molecular orbital (HOMO) corresponds to increase of optical absorption at 282 to $400 \mathrm{~nm}$ for AuNP size ranging from 4 to 10 $\mathrm{nm}[11]$.

For very small particles, LSPR signals are strongly damped and the principal mechanism of absorption is dominated by interband transitions [12]. For particles larger than $10 \mathrm{~nm}$, where it is still small compared to incident wavelengths of light, LSPR contributes to a sharp absorption peak at visible wavelengths [13]. For monodisperse AuNPs, extinction coefficients show a singular dipole LSPR peak when the particles are less than $100 \mathrm{~nm}$ in diameter. The peak wavelength redshifts with increasing intensity and profile widens as the particle size increases from $5 \mathrm{~nm}$ to $100 \mathrm{~nm}$. Similar to monodisperse AuNPs, Figure 2 shows single mode polydisperse particles with increasing mean particle diameter, $\langle\mathrm{d}\rangle$ at a fixed standard deviation of $40 \mathrm{~nm}$ have its absorption coefficient peaks sharply at dipole resonance when the particles are smaller than $\langle\mathrm{d}\rangle=20 \mathrm{~nm}$. The input of size distributions from figure 1(a) contributing to computation of all three coefficients are shown in figure 2 . When the particle sizes are within the dipole approximation limit - that its diameter compared to incoming wavelength at $d=1 / 10 \lambda$, the extinction coefficient is almost exclusively contributed by absorption. The peak intensity between interband transition and strong LSPR band at $521 \mathrm{~nm}$ are comparable at magnitude around $4 \times 10^{-12} \mathrm{~m}^{2}$. Since the plasmon resonance at this point was contributed almost completely by dipole plasmon oscillations, the extinction is transparent after $600 \mathrm{~nm}$. When particle size exceeds approximation regime, the magnitude of scattering term begin to increase. This contributes to overall broadening and increase of LSPR peak intensity up towards $18 \times 10^{-12} \mathrm{~m}^{2}$. At this point, dipole discrete approximation (DDA) computations will begin to deviate from spectrophotometry measurements and modification terms have to be introduced to account extinction contributed by large or irregular particles.

At $\langle\mathrm{d}\rangle=80 \mathrm{~nm}$, the intensity of scattering term became comparable to the absorption, which dampens at increasing particle size. The peak waist from scattering term contributes increase of extinction longer than $600 \mathrm{~nm}$. Both absorption and scattering peaks contribute to broadening of the LSPR band. When mean particle size reaches $\langle\mathrm{d}\rangle=120 \mathrm{~nm}$, sharp absorption peak almost completely damped while scattering begins to dominate. Compared to other PSDs, the extinction is at highest magnitude at this size and considerable broadening arisen from gradual appearance of higher mode plasmon oscillations. The LSPR peak width has red shifted close to $600 \mathrm{~nm}$. Beyond $\langle\mathrm{d}\rangle=120 \mathrm{~nm}$, the particles are considered as sub-micrometer scatterers where peak extinction intensity gradually decreases with larger particles. A secondary peak contributed by quadrupole plasmon oscillation becomes obvious at $\langle\mathrm{d}\rangle=200 \mathrm{~nm}$. At this particle sizes the ratio of extinction intensity towards the red-end of the spectrum becomes comparable with interband transitions. 

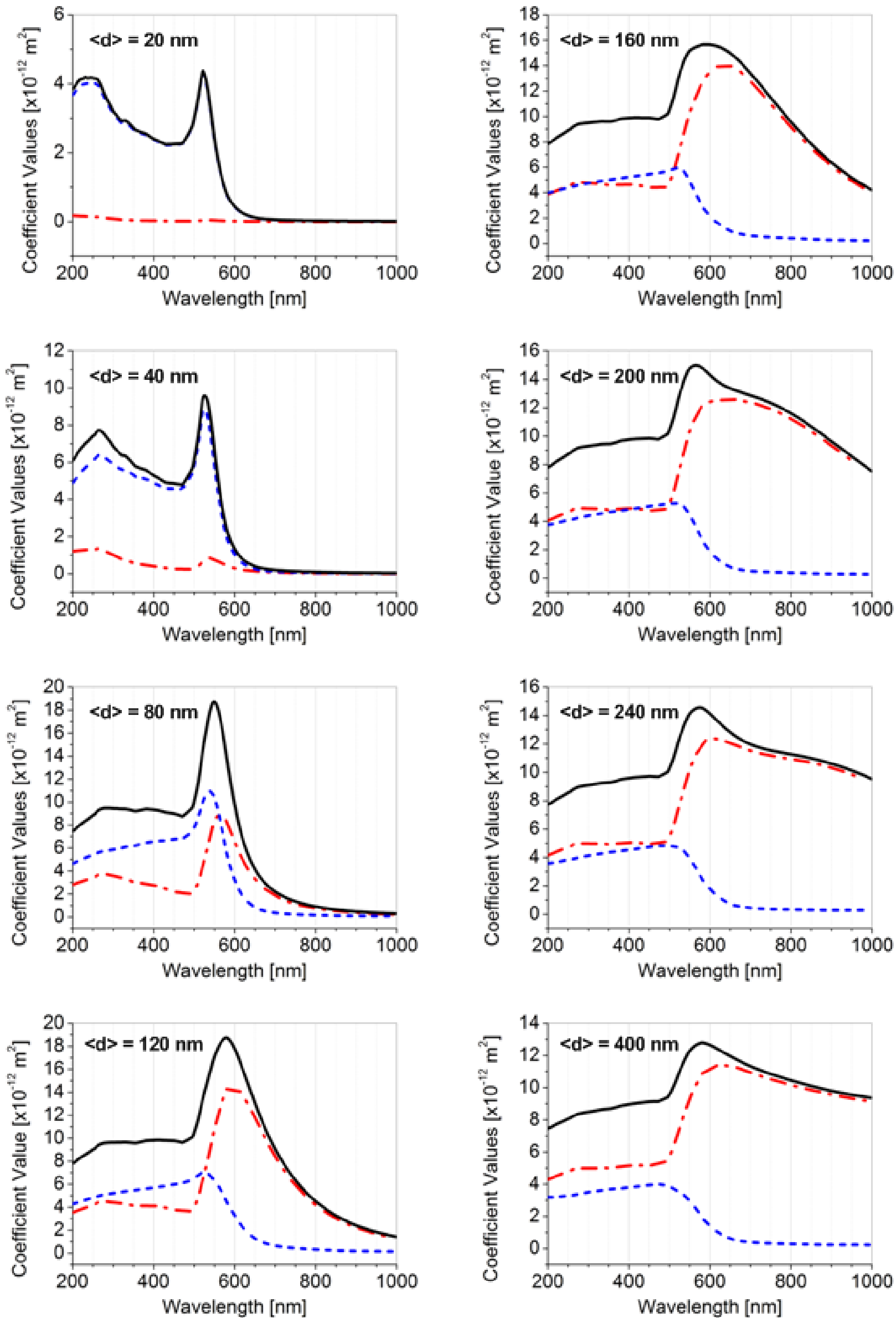

Fig. 2 Extinction (solid black), scattering (dot dashed red) and absorption (dotted blue) coefficients of polydisperse AuNP of increasing log-normal mode diameter, $<\mathrm{d}>$ all sets are produced with standard deviation of $40 \mathrm{~nm}$. 
(a)

(b)
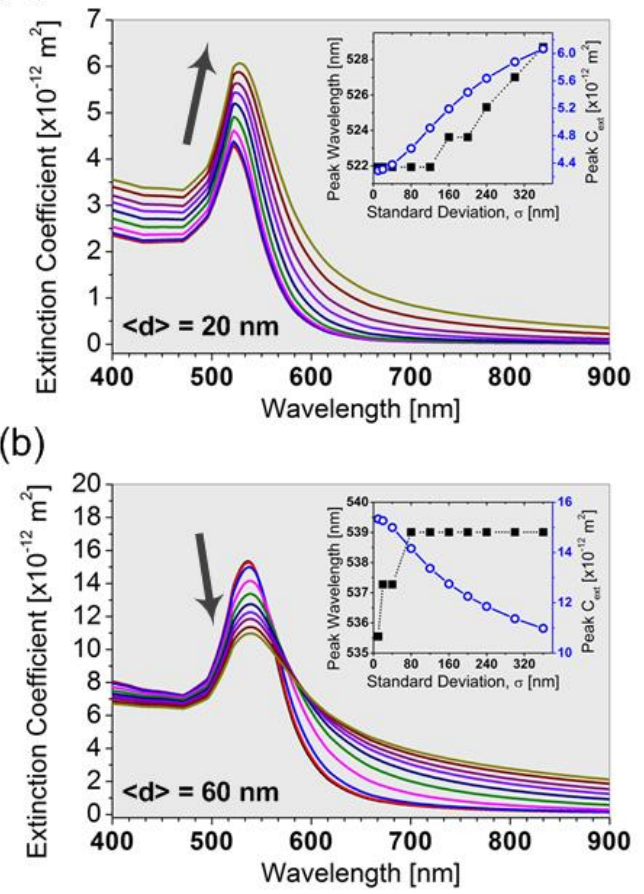

Fig. 3 (a) Simulated extinction coefficient spectra obtained by increasing standard deviation on particle sizes at $<\mathrm{d}>=20 \mathrm{~nm}$ and $(\mathbf{b})<\mathrm{d}>=60 \mathrm{~nm}$. The (insets) shows the corresponding shifts in LSPR peak wavelength and intensity values respectively.

In realistic sample conditions, increasing degree of polydispersity causes experimental extinction spectra to deviate from simulations even when average particle sizes falls within DDA regime [14]. The changes in spectral profile intensities and peak wavelengths depend heavily on mode particle sizes where increasing standard deviation on particle size of $\langle\mathrm{d}\rangle=20 \mathrm{~nm}$ within DDA approximation causes bandwide increase in coefficient magnitude. Broadening of extinction between 600 to 700 $\mathrm{nm}$ corresponds to scattering contributed by large particles. Increasing standard deviation on large particles of $\langle\mathrm{d}\rangle=60 \mathrm{~nm}$ however shows decrease in LSPR peak throughout interband transition wavelengths as dipole absorption is strongly damped, this is accompanied by rapid increase of extinction intensity towards the red end of the spectrum contributed by scattering from sub-micrometer particles. (a)

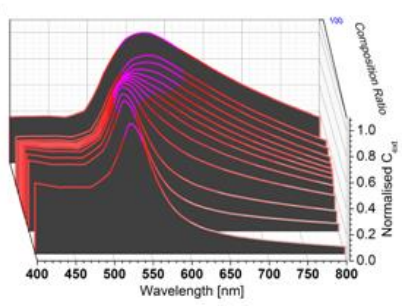

(b)

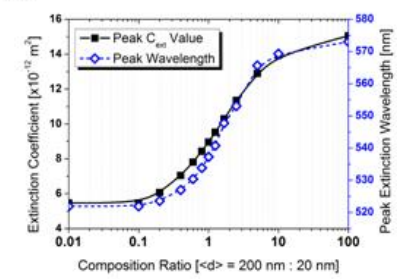

Fig. 4 (a) Normalised extinction coefficients for bimodal AuNPs of increasing composition ratio between particle sizes of $200 \mathrm{~nm}$ and 20 $\mathrm{nm}$ at standard deviation of $100 \mathrm{~nm}$. (b) The peak LSPR wavelength and intensities for non-normalised extinction coefficient values.

The LSPR peak wavelength also red-shifted more compared to earlier set of data. The results from figure 3 (a) and (b) shows the dynamics of extinction spectra expected from in-situ measurements. The size dependent LSPR band broadening implies the application of real-time particle sizing with unmodified DDA computation highly inaccurate, especially for the purpose of synthesis monitoring where high degree of polydispersity are expected. Following AuNP fragmentation growth model proposed by Pong et. al. [15] and Mikhlin et. al. [16], the colloid exhibit optical bandwide extinction shortly after reduction of gold (III) ions, which then narrows into a distinct LSPR peak at $532 \mathrm{~nm}$ for well formed $20 \mathrm{~nm}$ particles [17]. Simulation of extinction spectra based on bimodal size distribution in figure 1 (b) where diminishing composition ratio between two populations of $\left\langle\mathrm{d}_{200}\right\rangle=200 \mathrm{~nm}$ and $\left\langle\mathrm{d}_{20}\right\rangle=20 \mathrm{~nm}$ particles at standard deviation of $100 \mathrm{~nm}$ shows considerable narrowing of LSPR band as indicated by figure 4 (a), forming a distinct sharp peak. The peak wavelength blue-shifts to $540 \mathrm{~nm}$ as large particles disintegrate into the same number of stable small particles. In figure 4 (b) composition ratio $\gamma$, between the particle populations at 5 to 0.5 shows linear relationship in linear-log plot for both changes in peak extinction wavelength and intensity. Maximum extinction at interband transitions occurs at PSD ratio of $\gamma=1: 1$. If the total numbers of particles remain the same, the maximum extinction coefficient value is expected to decrease as monodisperse particles eventually emerge and replace larger particles.

Another important aspect on in-situ monitoring of colloidal AuNPs produced with heat-assisted citrate reduction methodologies [18-19] is the changes in extinction spectra caused by fluctuating refractive indices of boiling reactants. Considering input of water-dispersed AuNP with mean size typical to the yield of standard Turkevich protocol [20] at $<\mathrm{d}>=20 \mathrm{~nm}$ with $\sigma=40 \mathrm{~nm}$ produces strong extinction peak at $522 \mathrm{~nm}$ as illustrated in figure 5 (a). Increasing colloidal temperature at the present resolution does not shift the peak wavelength while changes on spectral intensity are significantly smaller compared to effects contributed by changes in particle size distribution. Assuming isochoric condition, the extinction coefficient decreased at absolute maximum of $4.5 \%$ with temperature increased from ambient to boiling environment; this correspond to maximum changes on peak extinction coefficient value at $\Delta \mathrm{C}_{\mathrm{ext}}=1.78 \times 10^{-13} \mathrm{~m}^{2}$. It should be noted that the physical extinction intensities depends on experimental constants such as path length and physical parameters such as particle densities which are subjected to dispersant viscosity, fluid dynamics and physicochemical behavior of the particles.

(a)

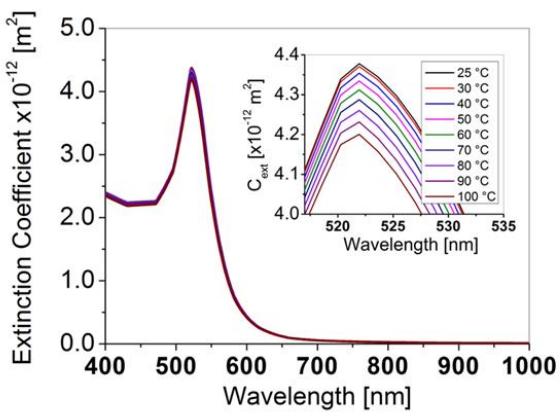

(b)

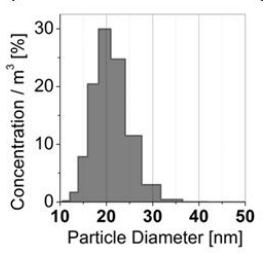

(c)

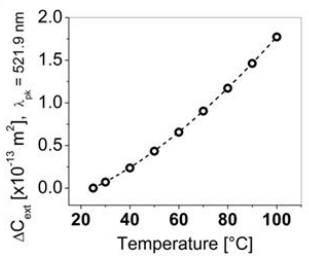

Fig. 5 (a) Extinction coefficient spectra produced by increasing temperature from $25^{\circ} \mathrm{C}$ to $100^{\circ} \mathrm{C}$. (Inset) magnified region on LSPR peak. (b) PSD used in computation. (c) Peak extinction values at increasing temperature.

\section{CONCLUSION}

Spectral characteristics on extinction coefficient based on increasing mean particle sizes, degree of polydispersity, ratio of bimodal PSD and changes in dispersant temperature are simulated from exact solution of Mie theory. It is clear that changes in colloidal temperature will not affect extinction coefficient as much compared to PSD dynamics if localized convection current can be avoided with careful experimental design. Changes on mean particle diameter affect LSPR peak wavelength greater than polydispersity. The latter decreases peak extinction coefficient for PSDs with mean diameter beyond dipole 
approximation regime. Diminishing population of large $\langle\mathrm{d}\rangle=200 \mathrm{~nm}$ particles in a bimodal size distribution replaced by emergence of smaller $\langle\mathrm{d}\rangle=20 \mathrm{~nm}$ particles will produce narrowing and blue-shift of LSPR band. These data shows promising extinction profile dynamics to describe colloidal state in-situ during synthesis.

\section{ACKNOWLEDGEMENTS}

This work was supported by Malaysian Ministry of Higher Education and Research University Grant of Universiti Teknologi Malaysia (flagship vote 00G79), MyPhd Scholarship (J. C. Chong), Malaysia.

\section{REFERENCES}

[1] S. M. Hong, Xiao Li, J. Nanomater. 2013 (2013) 9.

[2] M. H. Tu, T. Sun, K. T. V. Grattan, Sens. Actuators B: Chem 164 (2012) 43.

[3] X. H. Huang, P. K. Jain, I. H. El-Sayed, M. A. El-Sayed, Lasers Med. Sci. 23 (2008) 217

[4] G S. Eustis, M. A. El-Sayed, Chem. Soc. Rev. 35 (2006) 209

[5] G. Mie, Ann. Phys. 330 (1908) 377.
[6] C. F. Bohren, D. R. Huffman, Absorption and Scattering of Light by Small Particles, Wiley VCH-Verlag, 1998, p. 82.

[7] H. Du., Appl. Optics, 43 (2004) 9, 1951.

[8] P. B. Johnson, R. W. Christy, Phys. Rev. B. 6 (1972) 4370

[9] D. Segelstein, M. S. Thesis, Univ. Missouri, (1981).

[10] F. Hache, D. Ricard, C. Flytzanis, U. Kreibig, Appl. Phys. A, 47 (1988) 347.

[11] U. Kreibig, M. Vollmer, Optical Properties of Metal Clusters, SpringerVerlag Berlin, 1995, p. 532.

[12] J. Zheng, C. Zhou, M. X. Yu, J. B. Liu, Nanoscale, 14 (2012) 4073.

[13] X. D. Li, T. P. Chen, Y. Liu, K. C. Leong, Opt. Express, 22 (2014) 5124.

[14] G. Yin, S. Y. Wang, M. Xu, L. Y. Chen, J. Korean Phys. Soc. 49 (2006) 2108.

[15] B. K. Pong, H. I. Elim, J. X. Chong, W. Ji, B. L. Trout, J. Y. Lee, J. Phys. Chem. C. 111 (2007) 6281

[16] Y. Mikhlin, A. Karacharov, M. Likhatski, T. Podlipskaya, Y. Zubavichus, A. Veligzhanin, V. Zaikovski, J. Colloid Interface Sci. 362 (2015) 330.

[17] J. C. Chong, S. L. Lee, N. Bidin, Jurnal Teknologi, 78 3-2 (2016) 165.

[18] J. Kimling, M. Maier, B. Okenve, V. Kotaidis, H. Ballot, A. Plech, J. Phys. Chem. B. 110 (2006) 15700.

[19] G. Fren, Nature, 241 (1973) 20

[20] J. Turkevich, P. C. Stevenson, J. Hiller, Discuss. Farad. Soc. 11 (1951) 55. 